\title{
Results from the $G^{0}$ Forward Angle Measurement
}

\author{
J. Liu, for the $G^{0}$ Collaboration \\ Department of Physics, University of Maryland, College Park, MD 20742
}

\begin{abstract}
The results from the $G^{0}$ forward angle experiment are reported in this talk. The parityviolating asymmetry of elastic e-p scattering has been measured within the range of the fourmomentum transfer $\left(Q^{2}\right)$ from 0.12 to $1.0(\mathrm{GeV} / \mathrm{c})^{2}$, which yields linear combinations of the strange electric and magnetic form factors of the nucleon, $G_{E}^{s}+\eta G_{M}^{s}$, in the same $Q^{2}$ range. The $G^{0}$ results, combined with the measurements from other experiments, indicate that $G_{E}^{s}$ and $G_{M}^{s}$ are both likely non-zero.
\end{abstract}

Keywords: $G^{0}$, parity violating, strangeness, $G_{E}^{s}, G_{M}^{s}$

PACS: 13.60.-r, 13.60.Fz, 11.30.Er, 13.40.Gp, 14.20.Dh

The strange electric and magnetic form factors of the nucleon, $G_{E}^{S}$ and $G_{M}^{S}$, can be determined by combining the well-known electromagnetic (EM) form factors of the proton and the neutron, $G_{E, M}^{\gamma, p}$ and $G_{E, M}^{\gamma, n}$, with the less-measured neutral weak form factors of the proton, $G_{E, M}^{Z, p}[1]$ :

$$
G_{E, M}^{s}=\left(1-4 \sin ^{2} \theta_{W}\right) G_{E, M}^{\gamma, p}-G_{E, M}^{\gamma, n}-G_{E, M}^{Z, p} .
$$

Due to the participation of the neutral weak interaction, elastic e-p scattering carries a small parity violating (PV) asymmetry, which gives direct access to $G_{E, M}^{Z, p}$. The PV asymmetry can be expressed as

$$
A_{P V}=-\frac{G_{F} Q^{2}}{4 \sqrt{2} \pi \alpha} \frac{\varepsilon G_{E}^{\gamma}\left(G_{E}^{Z}+\eta G_{M}^{Z}\right)-\left(1-4 \sin ^{2} \theta_{W}\right) \varepsilon^{\prime} G_{M}^{\gamma} G_{A}^{e}}{\varepsilon\left(G_{E}^{\gamma}\right)^{2}+\tau\left(G_{M}^{\gamma}\right)^{2}},
$$

(with the "p" superscripts of the form factors suppressed) and

$$
\tau=\frac{Q^{2}}{4 M_{p}^{2}}, \varepsilon=\left(1+2\left(1+\tau \tan ^{2} \frac{\theta_{e}}{2}\right)\right)^{-1}, \varepsilon^{\prime}=\sqrt{\tau(1+\tau)\left(1-\varepsilon^{2}\right)} \text { and } \eta=\frac{\tau G_{M}^{\gamma}}{\varepsilon G_{E}^{\gamma}} \text {. }
$$

In Eqn. $2, G_{A}^{e}$ is the axial form factor of the proton as seen by an electron. If one treats both the EM and the axial form factors as known, a measurement of $A_{P V}$ determines $G_{E}^{Z}+\eta G_{M}^{Z}$, immediately leading to $G_{E}^{s}+\eta G_{M}^{s}$ using Eqn. 1 .

$G^{0}$ is an experiment at Jefferson lab making precise measurements of these PV asymmetries [2]. A longitudinally polarized electron beam with $\sim 73.7 \%$ polarization, $3.03 \mathrm{GeV}$ energy and $\sim 40 \mu \mathrm{A}$ current was used, with micro-bunches separated by $32 \mathrm{~ns}$. The helicity of the beam was alternated at $30 \mathrm{~Hz}$. The target was a $20 \mathrm{~cm}$ long, unpolarized liquid hydrogen target, located in the center of a superconducting

\footnotetext{
CP842, Particles and Nuclei,

Seventeenth International Conference on Particles and Nuclei

edited by P. D. Barnes, M. D. Cooper, R. A. Eisenstein, H. van Hecke, and G. J. Stephenson

(C) 2006 American Institute of Physics 0-7354-0338-4/06/\$23.00
}

306 
toroidal magnet. The scattered particles with positive charge were focused onto an array of scintillating detectors located at the focal plane of the spectrometer. These detectors covered the elastic $Q^{2}$ range of $0.12-1.0(\mathrm{GeV} / \mathrm{c})^{2}$. The particle identification $\left(\pi^{+}\right.$, inelastic and elastic protons) was made via their different time-of-flight. The electronics counted individual particles from which a time-of-flight histogram for each beam helicity state was constructed. The PV asymmetry is the relative difference of the detector yields between the two beam helicities.

Careful corrections had to be made to the raw asymmetries. The two most significant corrections were due to the contaminations of the leakage beam intended for other experimental halls (with different time structure), and the background underneath the elastic peak $[2,9]$. The final physics asymmetries as a function of $Q^{2}$ are plotted in Fig. 1. On each data point, the inner error bar is the statistical uncertainty, and the outer one is the combined statistical and the point-point systematic uncertainty. The upper gray band represents the global systematic uncertainty. The black curve is the nonvector-strange asymmetry, $A_{N V S}$, calculated by setting both $G_{E}^{s}$ and $G_{M}^{S}$ to zero, with the parameterizations of the nucleon EM form factors from [10]. $G_{E}^{s}+\eta G_{M}^{s}$, extracted from the difference between the measured asymmetries and $A_{N V S}$ in Fig. 1, is displayed in Fig. 2. We note that $\eta \sim 0.94 Q^{2}$ for $G^{0}$ forward angle kinematics. The inner and outer error bars on the data points, and the upper gray band share the same nature as those in Fig. 1. The lower light-gray band is the uncertainty due to the electroweak radiative corrections to the PV asymmetries. The degree to which the non-vector-strange line $\left(G_{E}^{s}+\eta G_{M}^{s}=0\right)$ would shift resulting from different EM form factor parameterizations is represented by the two dashed curves (pink: [11], green: [12]). Finally, a $\chi^{2}$ test based on the $G^{0}$ data, taking into account both the random and correlated errors in Fig. 2, disfavors the hypothesis of $G_{E}^{s}+\eta G_{M}^{s}=0$ with $89 \%$ confidence level.

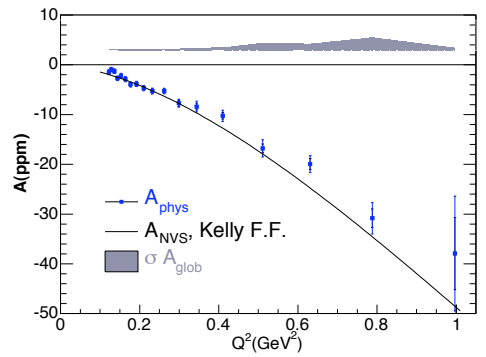

FIGURE 1. The physics asymmetry vs. $Q^{2}$ measured in the $G^{0}$ forward angle experiment. The black curve is the non-vector-strange asymmetry. See text for details.

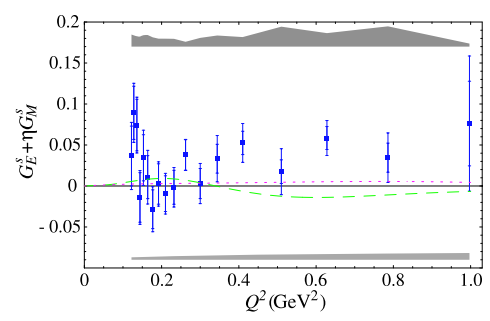

FIGURE 2. The extracted $G_{E}^{s}+\eta G_{M}^{s}$ vs. $Q^{2}$. The dashed lines shows the impact of different parametrization of the EM form factor of the nucleons. See text for details.

The $Q^{2}$ range of $G^{0}$ overlaps with other existing measurements at $0.1,0.23$ and $0.477(\mathrm{GeV} / \mathrm{c})^{2}$. The data at $Q^{2}=0.1(\mathrm{GeV} / \mathrm{c})^{2}$ from $G^{0}$ [2], SAMPLE [3], HAPPEx [7, 8] and PVA4 [5] are shown Fig. 3(a). All five measurements agree, and in combination they lead to $G_{M}^{s}=0.55 \pm 0.28$ ( $G_{E}^{s}$ is consistent with zero within precision), leading to an approximately $-10 \%$ contribution of strange quarks to the proton's magnetic moment at the $2 \sigma$ level. The $1 \sigma$ and $95 \%$ confidence contours are also drawn in the figure. 
The world data at $Q^{2}=0.23(\mathrm{GeV} / \mathrm{c})^{2}\left(G^{0}\right.$ [2] and PVA4 [4]) and $0.477(\mathrm{GeV} / \mathrm{c})^{2}$ $\left(G^{0}[2]\right.$ and HAPPEx [6]) are shown in Figs. 3(b) and 3(c), respectively. Although more measurements are needed at these two points to independently determine $G_{E}^{s}$ and $G_{M}^{s}$, the existing data are also in excellent agreement.

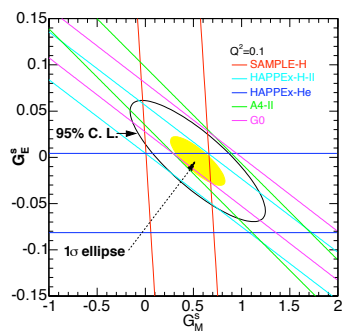

(a) $Q^{2}=0.1(\mathrm{GeV} / \mathrm{c})^{2}$

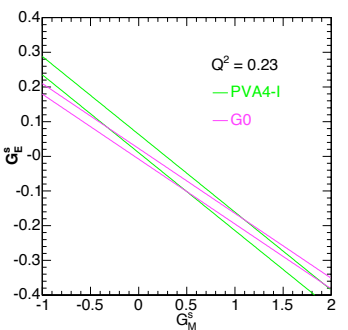

(b) $Q^{2}=0.23(\mathrm{GeV} / \mathrm{c})^{2}$

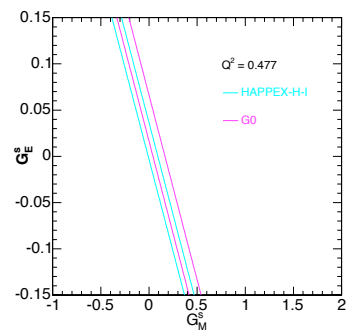

(c) $Q^{2}=0.477(\mathrm{GeV} / \mathrm{c})^{2}$

FIGURE 3. The constraints of the world data in $\left(G_{M}^{s}, G_{E}^{s}\right)$ space at three $Q^{2}$ s. See details in [9].

In Fig. 2, the positive data in the lowest $Q^{2}$ bins are in accord with a positive $G_{M}^{s}$ indicated by Fig. 3(a). The decreasing trend of the data around $0.2(\mathrm{GeV} / \mathrm{c})^{2}$ (with an increasing $\eta$ ) suggests that $G_{E}^{S}$ might be negative around this region. The results in Fig. 3(b) also provide some support to this speculation. There is a positive trend of $G_{E}^{s}+\eta G_{M}^{s}$ at higher $Q^{2}$, consistent with HAPPEx (Fig. 3(c)). The experiments together indicate that both $G_{E}^{s}$ and $G_{M}^{s}$ are non-zero and $Q^{2}$-dependent.

$G^{0}$ backward angle experiment will be started in 2006 to measure the PV asymmetries on both hydrogen and deuterium at $Q^{2}=0.23$ and $0.63(\mathrm{GeV} / \mathrm{c})^{2}$. The programs of PVA4 and HAPPEx are also continuing. These measurements will allow clean separations of $G_{E}^{S}$ and $G_{M}^{S}$ at different $Q^{2}$ values in the near future.

We gratefully acknowledge the support from our funding agencies (DOE, NSF, CNRS and NSERC) and the strong technical support from TJNAF and many other groups.

\section{REFERENCES}

1. D. H. Beck and R. D. McKeown, Ann. Rev. Nucl. Part. Sci. 51, 189 (2001)

2. D. S. Armstrong et al. $\left(G^{0}\right)$, Phys. Rev. Lett. 95, 092001 (2005).

3. D. T. Spayde et al. (SAMPLE), Phys. Letter. B 583, 79 (2004).

4. F. E. Mass et al. (PVA4), Phys. Rev. Letter. 93, 022002 (2004).

5. F. E. Mass et al. (PVA4), Phys. Rev. Letter. 94, 152001 (2005).

6. K. A. Aniol et al. (HAPPEx), Phys. Rev. C 69, 065501 (2004).

7. K. A. Aniol et al. (HAPPEx), nucl-ex/0506010.

8. K. A. Aniol et al. (HAPPEx), nucl-ex/0506011.

9. See, e.g., B. Guillon, Ph.D. Thesis, LPSC-Grenoble, October 2005.

10. J. J. Kelly, Phys. Rev. C 70, 068202 (2004).

11. J. Arrington, Phys. Rev. C 69, 022201(R) (2004).

12. J. Friedrich and T. Walcher, Eur. Phys. J. A 17, 607 (2003). 